DESY 08-030

LPT-ORSAY 08-31

PITHA 08/06

PSI-PR-08-03

March 18, 2008

\title{
Squark Cascade Decays to Charginos/Neutralinos: Gluon Radiation
}

\author{
R. Horsky ${ }^{1}$, M. Krämer ${ }^{1}$, A. Mück ${ }^{2}$, and P.M. Zerwas ${ }^{1,3,4}$ \\ ${ }^{1}$ Institut für Theoretische Physik E, RWTH Aachen, D-52056 Aachen, Germany \\ 2 Paul Scherrer Institut, Würenlingen und Villigen, Ch-5232 Villigen PSI, Switzerland \\ 3 Deutsches Elektronen-Synchrotron DESY, D-22603 Hamburg, Germany \\ ${ }^{4}$ Laboratoire de Physique Théorique, U. Paris-Sud, F-91405 Orsay, France
}

\begin{abstract}
The momentum spectrum and the polarization of charginos and neutralinos in squark decays are affected by gluon radiation in the decay process $\tilde{q} \rightarrow q \tilde{\chi}(g)$. We determine these corrections and study their impact on the $[q \ell]$ invariant mass distributions for leptonic $\tilde{\chi}$ decays. The higher-order corrections, though small in general, can be sizeable near pronounced edges of the final-state distributions.
\end{abstract}




\section{Introduction}

The properties of supersymmetric particles, colored and non-colored, can be studied at the LHC in cascade decays of squarks and gluinos, which have large production cross sections in high-energy hadron collisions. In particular, squarks that are lighter than gluinos decay exclusively to quarks and charginos or neutralinos, $\tilde{q} \rightarrow q \tilde{\chi}$. Subsequent decays of neutralinos into final states with two leptons are especially well suited to investigate properties of the supersymmetric spectrum. Invariant mass distributions of the final-state leptons and jets can be exploited, for example, for precision measurements of supersymmetric particle masses (see e.g. [1-4]). Moreover, correlations among the particles in the decay chains allow spin measurements of intermediate particles [5-8].

In the present article we concentrate on one aspect in the multitude of theoretical challenges involved in the description of supersymmetric cascade decays, i.e. the super-QCD (SQCD) radiative corrections at next-to-leading order (NLO) and their impact on energy distributions, polarization degrees, and on the shape of quark-lepton invariant mass distributions. Precise theoretical predictions for the shape of the distributions are required also at some distance away from kinematic edges and thresholds as to allow for sufficiently large, statistically significant event samples. Moreover, the shapes may be needed to resolve possible ambiguities in mass determinations from kinematic endpoints [9], they are crucial for the spin determination [5], and they allow to test models of supersymmetry breaking [10]. We investigate the impact of QCD radiation on the shape of distributions in NLO perturbation theory and derive, largely, analytical results which provide us with the proper understanding of the SQCD effects. Extending the Monte Carlo calculation of QCD effects in Ref. [11], we include gluon radiation off the final-state quarks. [Hadronic jet fragmentation which must finally be added is beyond the scope of the present study, but the effect can be estimated by means of QCD power corrections.]

We shall, for definiteness, focus on cascades of $L$-type squarks, $\tilde{q}_{L}$, of the first two generations without noticeable $L / R$ mixing and consider the decay chain

$$
\tilde{q}_{L} \rightarrow q \tilde{\chi}_{2}^{0} \rightarrow q \ell \tilde{\ell}_{R} \rightarrow q \ell \ell \tilde{\chi}_{1}^{0}
$$

which has been discussed in great detail in the literature for the SPS1a/a' benchmark points $[12,13]$. The neutralino $\tilde{\chi}_{1}^{0}$ is assumed to be the lightest supersymmetric particle (LSP) and thus is stable in R-parity conserving theories. Since the Born terms can be factored out, the NLO analysis applies, without modifications, to all neutralino and chargino decay chains of the same form. $R$-type squarks, $\tilde{q}_{R}$, decay in large parts of the supersymmetric parameter space, particularly at the benchmark points SPS1a/a', to the LSP, $\tilde{q}_{R} \rightarrow q \tilde{\chi}_{1}^{0}$, and do not develop cascades.

Spin correlations are important for the proper description of the cascade decay (1.1). Since only the gaugino components of the neutralino $\tilde{\chi}_{2}^{0}$ couple effectively to the squark/quark current if quark masses are neglected, the $\tilde{q}_{L}$ squarks decay to left-handedly polarized $q_{L}$ quarks. By angular momentum conservation also the neutralino $\tilde{\chi}_{2}^{0}$ is polarized left-handedly at the Born level. Gluon radiation in the 

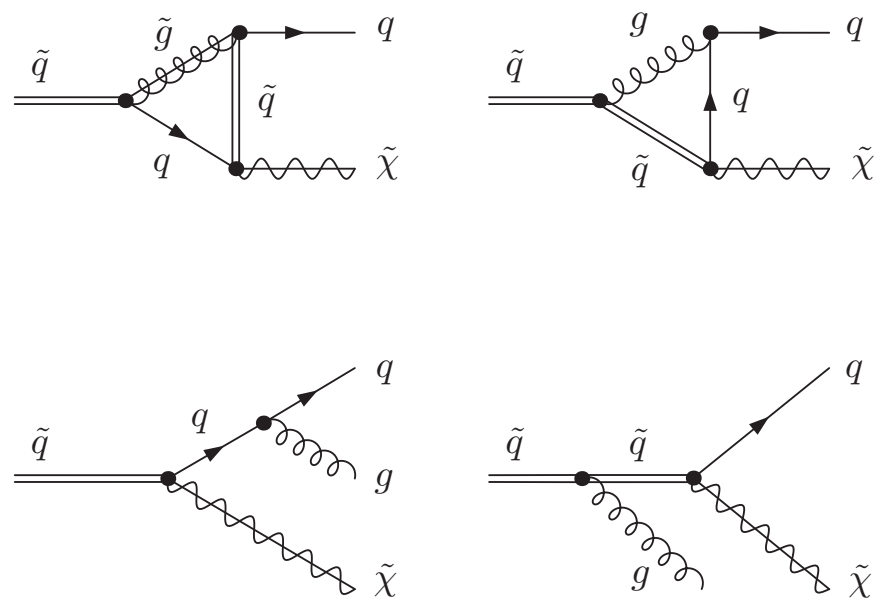

Figure 1: Generic Feynman diagrams for virtual and real super-QCD corrections to the decay $\tilde{q} \rightarrow q \tilde{\chi}$.

decay process leads to an admixture of right-handed neutralinos. The polarization is reflected in the energy and angular distributions of the decay products.

The paper is organized as follows. In section 2 we shall present the results for the NLO SQCD corrections to the decay $\tilde{q}_{L} \rightarrow q \tilde{\chi}$ and their impact on the $\tilde{\chi}$ energy distribution and polarization. The phenomenology of the complete $\tilde{q}_{L}$ decay chain (1.1), including the spin correlations, is discussed in section [3.1. In section 3.2 we analyze the decay chains in the broader framework of squark production at the LHC, including $R$-squarks and anti-squarks. We conclude in section 4 .

\section{Supersymmetric QCD corrections to the decay $\tilde{q} \rightarrow q \tilde{\chi}$}

Squark and gluino decays are affected by SQCD corrections. For their mutual decay modes, dominating if kinematically allowed, the NLO corrections were determined in Ref. [14]. The SQCD corrections to the partial widths for the electroweak squark decay channels,

$$
\tilde{q} \rightarrow q+\tilde{\chi}+(g)
$$

were calculated in Refs. [15-18]: Virtual gluon and gluino/squark exchanges renormalize the $q \tilde{q} \tilde{\chi}$ vertex and additional gluons are radiated in the final state, $c f$. Fig. 1.

Vertex corrections neither change the $\tilde{\chi}$ momentum nor its polarization state, but both are affected by gluon radiation. The $\tilde{\chi}$ line spectrum of the two-body decay becomes a continuous spectrum (section 2.1). Since in radiative decays the $\tilde{\chi}$ is not back-to-back anymore with the polarized zero-mass 
quark q, spin-flipped $\tilde{\chi}$ states are admixed (section 2.2). While the effect on the polarization is small, the overall impact of gluon radiation on the final-state distributions, particularly near edges, is quite significant, nota bene in view of the envisaged high-precision analyses of these cascade modes at the LHC.

\section{$2.1 \tilde{\chi}$ Energy distribution}

The Dalitz-plot distribution for the unpolarized decay $\tilde{q} \rightarrow q \tilde{\chi} g$, normalized to the Born-level decay width $\Gamma_{\mathrm{B}}$,

$$
\frac{1}{\Gamma_{\mathrm{B}}} \frac{d \Gamma}{d x_{q} d x_{g}}=\frac{4}{3} \frac{\alpha_{\mathrm{s}}}{\pi} \frac{1}{(1-\kappa)^{2}}\left[\frac{2-2 \kappa\left(1+x_{q}\right)-x_{g}\left(1+\kappa+x_{q}\right)}{2 x_{g}\left(1+\kappa-x_{\tilde{\chi}}\right)}-\frac{(1-\kappa)-x_{g}}{x_{g}^{2}}\right],
$$

is expressed by $x_{q, g, \tilde{\chi}}=2 E_{q, g, \tilde{\chi}} / M_{\tilde{q}}$, where $M_{\tilde{q}}$ is the squark mass and $E_{q, g, \tilde{\chi}}$ are the energies of the quark, the gluon, and the $\tilde{\chi}$ in the squark rest frame with $x_{\tilde{\chi}}+x_{q}+x_{g}=2$ and $2 \sqrt{\kappa} \leq x_{\tilde{\chi}} \leq 1+\kappa$. A useful abbreviation is the mass ratio $\kappa=M_{\tilde{\chi}}^{2} / M_{\tilde{q}}^{2}$. The partial width in Born approximation, Refs. [16, 17],

$$
\Gamma_{B}=\frac{\alpha}{4} M_{\tilde{q}}(1-\kappa)^{2} f_{\tilde{q} \tilde{\chi}}^{2}
$$

depends on the chargino and neutralino mixing parameters $[19,20]$ in the coefficients $f_{\tilde{q} \tilde{\chi}}$. Gluon radiation collinear with the final-state quark is described by the first term in Eq. (2.2) since $1+\kappa-x_{\tilde{\chi}}=M_{q g}^{2} / M_{\tilde{q}}^{2}$, where $M_{q g}$ is the $[q g]$ invariant mass. The second term damps the infrared singularity.

Gluon radiation reduces the energy $x_{\tilde{\chi}}$ of the $\tilde{\chi}$ according to the distribution

$$
\frac{1}{\Gamma_{\mathrm{B}}} \frac{d \Gamma}{d x_{\tilde{\chi}}}=\frac{4}{3} \frac{\alpha_{\mathrm{S}}}{\pi} \frac{1}{(1-\kappa)^{2}} \frac{1}{1+\kappa-x_{\tilde{\chi}}}\left[(1-\kappa)\left(2-x_{\tilde{\chi}}\right) \log \left(\frac{2-x_{\tilde{\chi}}+p_{\tilde{\chi}}}{2-x_{\tilde{\chi}}-p_{\tilde{\chi}}}\right)+\frac{1}{4} p_{\tilde{\chi}}\left(x_{\tilde{\chi}}-8+6 \kappa\right)\right],
$$

where $p_{\tilde{\chi}}=\sqrt{x_{\tilde{\chi}}^{2}-4 \kappa}$ denotes the scaled neutralino momentum in the squark rest frame.

Collinear and infrared gluon emission generate singularities at the maximum $\tilde{\chi}$ energy $x_{\tilde{\chi}} \rightarrow x_{\tilde{\chi}}^{\max }=$ $1+\kappa$. The leading contribution in $\delta=x_{\tilde{\chi}}^{\max }-x_{\tilde{\chi}}$ can be resummed by applying techniques analogous to thrust distributions [21-23] or lepto-quark decays [24]:

$$
\begin{aligned}
\frac{1}{\Gamma_{\mathrm{B}}} \frac{d \Gamma}{d x_{\tilde{\chi}}} & \simeq \frac{4}{3} \frac{\alpha_{\mathrm{s}}}{\pi} \frac{1}{\delta}\left[\log \frac{1}{\delta}+c\right] \\
& \Rightarrow \frac{4}{3} \frac{\alpha_{\mathrm{s}}}{\pi} \frac{1}{\delta}\left[\log \frac{1}{\delta}+c\right] \exp \left[-\frac{4}{3} \frac{\alpha_{\mathrm{s}}}{\pi}\left(\frac{1}{2} \log ^{2} \frac{1}{\delta}+c \log \frac{1}{\delta}\right)\right]
\end{aligned}
$$

with the sub-leading coefficient

$$
c=-\frac{7}{4}+\frac{\log \left[(1-\kappa)^{2}\right]}{(1-\kappa)^{2}} .
$$

Multiple gluon emission bends the distribution to zero at the kinematical boundary, corresponding to the Sudakov suppression of a final state without any gluons. Depending in detail on the effective value of $\alpha_{\mathrm{s}}$, the turn-over point is very close to the maximum of $x_{\tilde{\chi}}$.

The $\tilde{\chi}$ energy distribution is displayed in Fig. 2(a) for $L$-squark decays to $\tilde{\chi}_{2}^{0}$, with parameters adopted from SPS1a' i.e. $\kappa=0.108$ ) and the QCD coupling set to $\alpha_{\mathrm{S}}\left(M_{\tilde{q}}\right)=0.093$. 


\section{$2.2 \tilde{\chi}$ Polarization vector}

Gluon radiation at NLO also affects the $\tilde{\chi}$ polarization. At the Born level the $\tilde{\chi}$ polarization in $\tilde{q}_{L}$ decays must be left-handed. While the $\tilde{\chi}$ spin does not flip for collinear and infrared gluon emission, because the intermediate left-chiral quark is effectively on-shell, the $\tilde{\chi}$ spin can flip for non-collinear/non-infrared final-state configurations, with the angular momentum balanced by the emitted hard gluon.

The polarization vector $\overrightarrow{\mathcal{P}}[\tilde{\chi}]$ is defined in the $\tilde{\chi}$ rest frame. The $x$-axis is chosen as the $\tilde{\chi}$ flight direction in the $\tilde{q}$ rest frame, the $[x, y]$-plane is identified with the decay plane, the $y$-axis pointing to the half-plane of shortest rotation from the $\tilde{\chi}$ to the $q$ momentum vector. The polarization vector lies within the decay plane, with components parallel, $\mathcal{P}_{\|}=\mathcal{P}_{x}$, and transverse, $\mathcal{P}_{\perp}=\mathcal{P}_{y}$, to the $\tilde{\chi}$ flight direction while the perpendicular polarization component vanishes $\left(\mathcal{P}_{z}=0\right)$.

The two components of the polarization vector for fixed $x_{\tilde{\chi}}$ can be cast into the form

$$
\begin{gathered}
\mathcal{P}_{\|}\left(x_{\tilde{\chi}}\right)=\frac{1}{4} \frac{1}{(1-\kappa)^{2}} \frac{1}{\left(1+\kappa-x_{\tilde{\chi}}\right) p_{\tilde{\chi}}}\left[4\left(2-x_{\tilde{\chi}}\right)\left(\kappa\left[4-x_{\tilde{\chi}}\right]-x_{\tilde{\chi}}\right) \log \left(\frac{2-x_{\tilde{\chi}}+p_{\tilde{\chi}}}{2-x_{\tilde{\chi}}-p_{\tilde{\chi}}}\right)+\right. \\
\left.\left(\left[8-x_{\tilde{\chi}}\right] x_{\tilde{\chi}}-4 \kappa\left[7-2 x_{\tilde{\chi}}\right]\right) p_{\tilde{\chi}}\right] / \mathcal{N}, \\
\mathcal{P}_{\perp}\left(x_{\tilde{\chi}}\right)=\frac{\pi}{8} \frac{1}{(1-\kappa)^{2}} \frac{\sqrt{\kappa}}{\sqrt{1+\kappa-x_{\tilde{\chi}}} p_{\tilde{\chi}}}\left[32+20 \kappa-16 \sqrt{1+\kappa-x_{\tilde{\chi}}}\left(2-x_{\tilde{\chi}}\right)-x_{\tilde{\chi}}\left(32-3 x_{\tilde{\chi}}\right)\right] / \mathcal{N} .
\end{gathered}
$$

The normalization factor $\mathcal{N}$ denotes the distribution $\left(\frac{4}{3} \frac{\alpha_{\mathrm{s}}}{\pi} \Gamma_{B}\right)^{-1} d \Gamma / d x_{\tilde{\chi}}$ at the point $x_{\tilde{\chi}}$. For fixed $x_{\tilde{\chi}}<x_{\tilde{\chi}}^{\max }$ the polarization vector is independent of $\alpha_{\mathrm{s}}$ to leading order and depends only on the radiation dynamics. In the infrared and collinear limits of gluon radiation, $x_{\tilde{\chi}} \rightarrow x_{\tilde{\chi}}^{\max }$, the Born values are approached again,

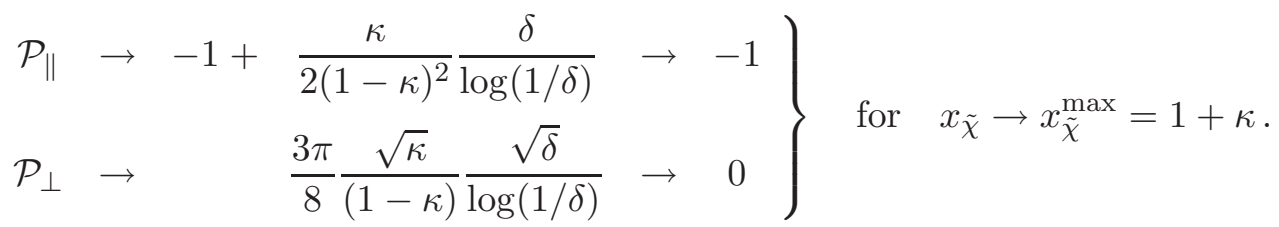

In the opposite limit, $x_{\tilde{\chi}} \rightarrow x_{\tilde{\chi}}^{\min }=2 \sqrt{\kappa}$, the polarization components approach the values $\mathcal{P}_{\|} \rightarrow 0$ and $\mathcal{P}_{\perp} \rightarrow-\pi / 4$. The two components of the polarization vector are displayed in Fig. 2(b), again for the mass pattern of the SPS1a' scenario $(\kappa=0.108)$.

\subsection{Average $\tilde{\chi}$ energy and polarization}

The values of the average energy and the polarization components of $\tilde{\chi}$ for the inclusive NLO prediction are obtained by integrating over the Dalitz plot and including the 2-body decay limit. Both, the QCD corrected 2-body and the 3-body part of the calculation are infrared divergent. Analogously to the methods employed in Refs. $[16,17]$, small quark and gluon masses are introduced to regularize 
a) $\tilde{\chi}$ Energy distribution $\left(x_{\tilde{\chi}}^{\max }-x_{\tilde{\chi}}\right) \Gamma_{\mathrm{B}}^{-1} \mathrm{~d} \Gamma / \mathrm{d} x_{\tilde{\chi}}$

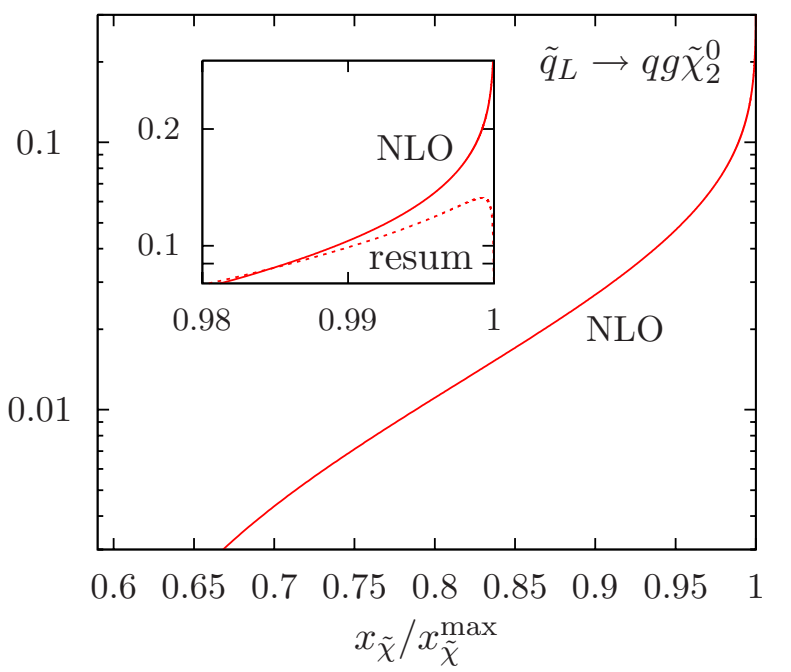

b) $\tilde{\chi}$ Polarization

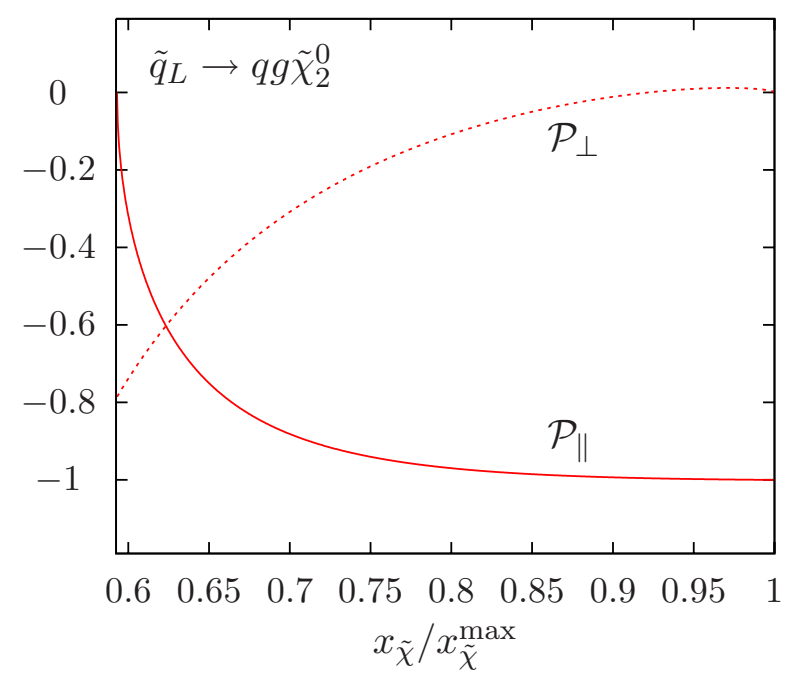

Figure 2: (a) The differential decay rate for $\tilde{q}_{L} \rightarrow q g \tilde{\chi}_{2}^{0}$ as a function of the $\tilde{\chi}_{2}^{0}$ energy $x_{\tilde{\chi}}$ at the benchmark point SPS1a' $\left(M_{\tilde{\chi}} / M_{\tilde{q}}=0.329\right)$. The curve labeled "resum" shows the damping near $x_{\tilde{\chi}}^{\max }$ from multiple gluon radiation according to Eq. (2.5). (b) The longitudinal and transverse polarization of the neutralino $\tilde{\chi}_{2}^{0}$.

the singularities. It is sufficient to know the regularized form of the different contributions for the unpolarized case. Since the polarization effects are not infrared-sensitive, the only divergent integrals are those already encountered in the unpolarized results. The averaged observables $\left\langle x_{\tilde{\chi}}\right\rangle,\left\langle\mathcal{P}_{\|}\right\rangle$, and $\left\langle\mathcal{P}_{\perp}\right\rangle$ globally reflect the deviation from the Born prediction to order $\alpha_{\mathrm{s}}$, and they are given by

$$
\left\langle x_{\tilde{\chi}}\right\rangle=x_{\tilde{\chi}}^{\max }-\frac{1}{9} \frac{\alpha_{\mathrm{s}}}{\pi} \frac{1}{(1-\kappa)^{2}}\left[(1-\kappa)(4-\kappa[17-7 \kappa])-6(2-\kappa) \kappa^{2} \log \kappa\right]
$$

and

$$
\begin{aligned}
&\left\langle\mathcal{P}_{\|}\right\rangle=-1-\frac{1}{9} \frac{\alpha_{\mathrm{S}}}{\pi} \frac{1}{(1-\kappa)^{2}}[ 4 \pi^{2}(\kappa-3) \kappa-48(\kappa-3) \kappa \operatorname{Li}_{2}(\sqrt{\kappa})+12(1-\kappa[8-3 \kappa]) \operatorname{Li}_{2}(\kappa) \\
&-3 \sqrt{\kappa}(22[1+\kappa]-\sqrt{\kappa}[45-\kappa])+6(1-\kappa)(11-3 \kappa) \log (\sqrt{\kappa}+1) \\
&\left.+3\left(4[1-\kappa]^{2} \log (1-\kappa)+\kappa[4-3 \kappa]\right) \log \kappa\right], \\
&\left\langle\mathcal{P}_{\perp}\right\rangle=\frac{2 \alpha_{\mathrm{s}}}{3} \frac{\sqrt{\kappa}}{(1-\kappa)^{2}}[4+4 \log \kappa-4 \kappa-(1+\sqrt{\kappa})(15-\kappa) \mathrm{E}-2(\kappa-7 \sqrt{\kappa}-8) \mathrm{K}] .
\end{aligned}
$$

The function $\mathrm{Li}_{2}$ is the usual dilogarithm while $\mathrm{K}$ and $\mathrm{E}$ abbreviate the complete elliptic integrals of the first and second kind 1 , respectively, evaluated for the argument $[(1-\sqrt{\kappa}) /(1+\sqrt{\kappa})]^{2}$.

For the reference point $\operatorname{SPS}^{\prime} \mathrm{a}^{\prime}(\kappa=0.108)$ adopted in the numerical analyses of the next section,

\footnotetext{
${ }^{1}$ The integrals are defined as $K(z)=\int_{0}^{\pi / 2}\left(1-z \sin ^{2} x\right)^{-1 / 2} d x$ and $E(z)=\int_{0}^{\pi / 2}\left(1-z \sin ^{2} x\right)^{1 / 2} d x$.
} 
the mass-dependent coefficients of the $\mathcal{O}\left(\alpha_{\mathrm{s}}\right)$ contributions take values

$$
\left\langle x_{\tilde{\chi}}\right\rangle=x_{\tilde{\chi}}^{\max }-0.32 \frac{\alpha_{\mathrm{s}}}{\pi},
$$

and

$$
\left\langle\mathcal{P}_{\|}\right\rangle=-1+0.02 \frac{\alpha_{\mathrm{s}}}{\pi}, \quad\left\langle\mathcal{P}_{\perp}\right\rangle=+0.08 \frac{\alpha_{\mathrm{s}}}{3} .
$$

The NLO SQCD effect on the average longitudinal component of the $\tilde{\chi}$ polarization vector, $\left\langle\mathcal{P}_{\|}\right\rangle$, is very small. This follows from the fact that in the collinear and infrared regions, in which the density of the Dalitz plot is maximal, the deviation of the polarization vector from the Born approximation approaches zero. The average perpendicular polarization $\left\langle\mathcal{P}_{\perp}\right\rangle$ is positive, dominated by the contributions from the region of large $\tilde{\chi}$ energies.

\section{Phenomenological results}

\subsection{The $\tilde{q}_{L}$ decay chain}

For parameter points like SPS1a/1a', the decays to lepton/slepton pairs of the first two generations,

$$
\tilde{\chi}_{2}^{0} \rightarrow \ell_{R}^{+}+\tilde{\ell}_{R}^{-} \text {and } \ell_{R}^{-}+\tilde{\ell}_{R}^{+}
$$

are ideal spin analyzers [ $R$ denoting right chirality] since the decays into the $L$-type state $\tilde{\ell}_{L}^{ \pm}$are kinematically forbidden, and $L / R$ slepton mixing as well as the $\tilde{\chi}_{2}^{0}$ higgsino component are suppressed. [Small corrections due to non-zero lepton masses can easily be included.]

For the sake of clarity, we first focus on these $\ell_{R}^{+} \tilde{\ell}_{R}^{-}$decay modes of the neutralino. Since the positron $\ell_{R}^{+}$is left-handedly polarized, it will be emitted preferentially anti-parallel to the $\tilde{\chi}$ spin direction,

$$
\frac{1}{\Gamma} \frac{d \Gamma}{d \cos \theta_{s}}=\frac{1}{2}\left(1-\mathcal{P}[\tilde{\chi}] \cos \theta_{s}\right)
$$

where $\theta_{s}$ is the angle between the $\tilde{\chi}$ spin vector and the $\ell^{+} 3$-momentum, and $\mathcal{P}[\tilde{\chi}]$ the $\tilde{\chi}$ degree of polarization. The angular correlation Eq. (3.2) gives rise to an increase of the invariant mass of the $\left[q \ell^{+}\right]$pair in the decay chain

$$
\tilde{q}_{L} \rightarrow q+\tilde{\chi}_{2}^{0}+(g) \rightarrow q+\ell^{+}+\tilde{\ell}_{R}^{-}+(g)
$$

compared with the isotropic distribution. [In this cascade the lepton $\ell$ is generally termed "near lepton" as opposed to the "far lepton" emitted in the subsequent slepton decay $\tilde{\ell} \rightarrow \ell \tilde{\chi}_{1}^{0}$, which will be included later.]

We have implemented the NLO results presented above in a flexible parton level Monte-Carlo program to calculate arbitrary differential distributions. Both phase-space slicing and subtraction methods have been employed to allow for internal cross checks. 
In the phase-space slicing approach, approximate real radiation matrix elements are integrated analytically in the soft/collinear regions using mass regularization. After adding the mass-regularized virtual corrections, the result is finite and the regulating quark and gluon masses can be set to zero. The hard gluon region is integrated by standard Monte-Carlo techniques.

Alternatively, a subtraction method has been applied which is particularly suited to construct NLO parton-level Monte-Carlo programs. In the present simple case the entire 3-particle differential decay width can be used as subtraction term. For the observables $\mathcal{O}$ which we consider, the expression in NLO can be cast in the form

$$
\langle\mathcal{O}\rangle_{\mathrm{NLO}}=\int_{3} d \hat{\Gamma}_{3}^{R}\left[\mathcal{O}_{3}-\mathcal{O}_{2}\right]+\int_{2}\left[d \hat{\Gamma}_{2}^{V}+\int_{1} d \hat{\Gamma}_{3}^{R}\right] \mathcal{O}_{2}
$$

Here, $d \hat{\Gamma}_{3}^{R}$ is the differential 3-parton decay width, $d \hat{\Gamma}_{2}^{V}$ the vertex-corrected 2-parton decay width, both normalized to the NLO width $\Gamma$. Moreover, $\mathcal{O}_{n}$ denotes the observable $\mathcal{O}$ calculated for either $n=3$ or $n=2$ parton final states. The projection from the $n+1$ onto the $n$ particle phase space is straightforward: at any given 3-body phase-space point, $\mathcal{O}_{2}$ is evaluated for a neutralino momentum which shares the direction of flight with the neutralino momentum of the 3-body phase-space point. The simple 2body kinematics fixes the neutralino and quark momenta completely so that $\mathcal{O}_{2}$ can be calculated unambiguously. The first integral in Eq. (3.4) is finite by construction and can be integrated by MonteCarlo techniques. The second and the third integral can be evaluated analytically [in mass-regulated form to isolate the infrared/collinear divergences, which cancel in the sum of the virtual contribution and the integrated subtraction term]. Since polarization effects are not infrared sensitive, it is sufficient to use the unpolarized differential decay width $d \hat{\Gamma}_{3}^{R}$ as a subtraction term even for observables including spin correlations. In this case, $\mathcal{O}_{2}$ is evaluated with the tree-level polarization vector, which provides the correct limit of the 3-body kinematics in the soft/collinear regions. The results derived by using the subtraction method agree with those obtained from phase-space slicing.

When gluon radiation is switched on, the $\left[q \ell^{+}\right]$invariant mass distribution is softened. To quantify this effect, the $q$-jet must be defined properly in an infrared-safe way. We will combine the $q$ and $g$ partons in the collinear and infrared regimes to a single $\hat{q}$-jet if their scaled invariant mass $y=M^{2}[\hat{q}=q g] / M_{\tilde{q}}^{2}$ is less than $y_{\mathrm{c}}=0.01$. For events with non-collinear hard gluon emission we identify $\hat{q}$ with the leading parton jet, i.e. the jet with the highest energy in the squark rest frame, either $q$ or, rarely, $g$.

The invariant mass distributions $M^{2}\left[\hat{q} \ell^{ \pm}\right]$are displayed as solid curves in Fig. 3, In the upper left panel the distribution of the positively charged "near" leptons, produced in $\tilde{\chi}_{2}^{0}$ decays, is shown. The dashed curve represents the invariant mass distribution at the Born level for comparison. The peak of the distribution at high invariant mass is more pronounced than the triangular-shaped tree-level distribution with spin correlations ignored [25]. As expected, the NLO corrections lead to the transfer of events to smaller invariant masses compared to the tree-level prediction.

The transition from the 1 -jet to the 2 -jet class of events gives rise to the tiny kink in the $\left[\hat{q} \ell_{\text {near }}^{+}\right]$ 
Jet-lepton invariant mass $\mathrm{d} \sigma / \mathrm{d} M_{\hat{q} l}[\mathrm{fb} / \mathrm{GeV}]$
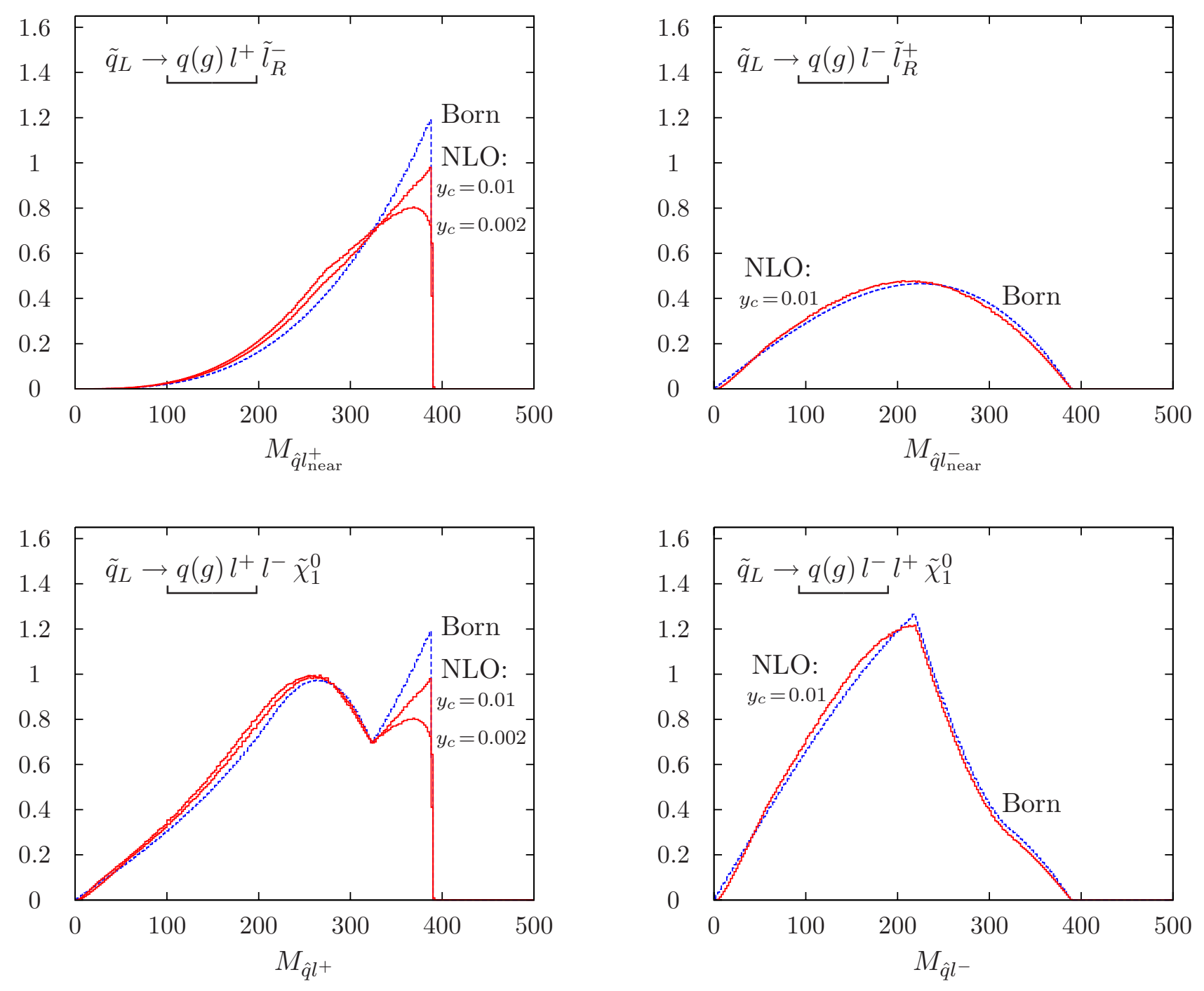

Figure 3: The $\left[\hat{q} l^{ \pm}\right]$invariant mass distributions for positively (left panels) and negatively (right panels) charged leptons is shown at SPS1a' [specifically for $\tilde{u}_{L}$ masses and first and second generation leptons with vanishing masses]. The upper panels include only "near" leptons while the lower panels display the sum of "near" and "far" leptons. The NLO predictions are represented by the solid lines for $y$-cut parameters $y_{\mathrm{c}}=0.01$ and 0.002, the Born result [approximately equivalent to NLO with $\left.y_{\mathrm{c}} \gtrsim 0.05\right]$ by the dashed line. 
distribution in Fig. 3. Varying the experimentally defined jet-resolution parameter $y_{\mathrm{c}}$ reproduces anticipated modifications of the mass distributions: the distribution is softened for smaller $y_{\mathrm{c}}$, and the peak rounded off, exemplified in Fig. 3 for $y_{\mathrm{c}}=0.002$, as a larger fraction of events with respect to the default choice $y_{\mathrm{c}}=0.01$ moves from the 1-jet to the 2-jet class. For larger $y_{\mathrm{c}}$ the distribution is sharpened, moving back towards the Born distribution, as more events are attributed to the 1-jet class. The NLO distribution becomes almost identical to the LO prediction for $y_{\mathrm{c}} \gtrsim 0.05$. Quite similar results for the invariant mass distributions are found when using a cone algorithm [26] defined in the squark rest frame.

Parallel to the $\tilde{\chi}$ energy distribution, exponential Sudakov damping of the invariant $\left[\hat{q} \ell^{+}\right]$mass distribution is expected in a margin of one percent below the pronounced kinematical edge when multiple gluon radiation is switched on. Likewise, hadronic jet fragmentation will give rise to smearing effects, generally accounted for, see e.g. Ref. [27], by shifting the perturbative prediction of the distribution downwards by a relative amount of approximately $1 \mathrm{GeV} / M_{\tilde{q}}$. For squarks decaying through electroweak channels, as at the present reference point, non-zero width effects $\propto \Gamma_{\tilde{q}} / M_{\tilde{q}}$ are also limited to about one percent, on both sides of the kinematical edge, though 2 These effects will round off the peak of the distribution at the edge for $y_{\mathrm{c}} \gtrsim 0.01$, with the overall impact limited however to a margin at the onepercent level for squark masses in excess of $500 \mathrm{GeV}$. With details depending on the jet definition, the modification will qualitatively be similar to the thrust distribution in jet analyses of $e^{+} e^{-}$annihilation at high energies [27]. The explicit Monte Carlo simulation of QCD effects in Ref. [11] includes only gluon radiation off the squarks which turns out, as expected, to have a very small effect on the shape of the distributions.

The experimentally observed lepton $\ell^{+}$may also be the decay product of the slepton in the chain 3 $\tilde{\chi}_{2}^{0} \rightarrow \ell_{R}^{-} \tilde{\ell}_{R}^{+} \rightarrow \ell_{R}^{-} \ell_{R}^{+} \tilde{\chi}_{1}^{0}$. For this "far" lepton $\ell^{+}$, the daughter of a spinless parent, the spin characteristics in the distributions are largely washed out and only the average energy reduction of the slepton due to spin correlations in the primary neutralino decay is effective.

Adding up the contributions from "near" and "far" $\ell^{+}$leptons, which cannot be separated experimentally on an event-by-event basis, we arrive at the distributions displayed in the lower left panel of Fig. 3. Due to the rather small mass difference between the selectron/smuon and the LSP $\tilde{\chi}_{1}^{0}$ in SPS1a', the lepton from the slepton decay is relatively low-energetic. Thus the "far" lepton adds to the distribution only at low invariant masses and the signature of the "near" lepton is still significant at high invariant masses.

If, instead, negatively charged leptons are observed the shapes of the two corresponding distributions are rather different. Since the negatively charged leptons are right-handedly polarized the sign in Eq. (3.2) flips and the "near" $\ell^{-}$is preferentially emitted parallel to the $q$-jet, in contrast to the antiparallel emission of positively charged leptons. The energy of the negatively charged lepton $\ell^{-}$is

\footnotetext{
${ }^{2}$ The accuracy of the narrow-width approximation for cascade decays has been addressed in Ref. [28].

${ }^{3}$ Electroweak radiative corrections to the decay $\tilde{\chi}_{2}^{0} \rightarrow l^{+} l^{-} \tilde{\chi}_{1}^{0}$ have been presented in Ref. [29].
} 


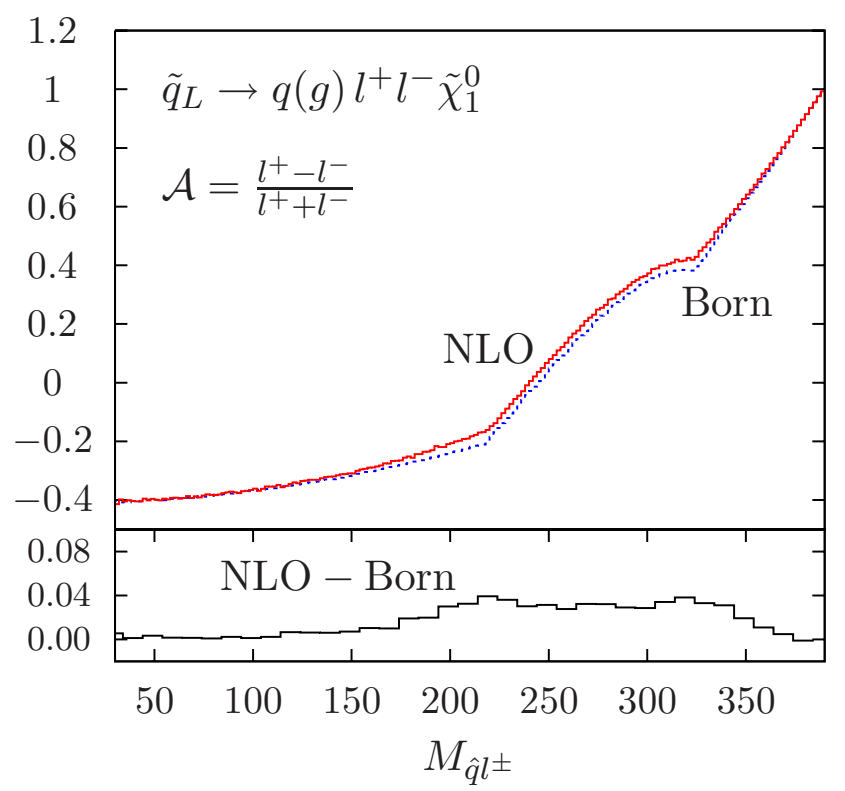

Figure 4: The asymmetry Eq. (3.5) as a function of the $\left[\hat{q} l^{ \pm}\right]$invariant mass at SPS1a for $\tilde{q}_{L}$ decays [specifically for $\tilde{u}_{L}$ masses] into first and second generation lepton pairs (no anti-squark decays included).

therefore, on average, reduced in the squark rest frame for polarized $\tilde{\chi}_{2}^{0}$ decays. For both reasons, the $\left[\hat{q} \ell^{-}\right]$invariant mass is reduced on average compared to the prediction without spin correlations [25]. The reduction is slightly enhanced by gluon emission as demonstrated in the upper right panel of Fig. 3. The distribution for the sum of "near" and "far" $\ell^{-}$leptons is shown in the lower right panel. Its shape can easily be distinguished from the $\ell^{+}$results at large invariant masses.

Spin correlations are clearly important for the description of the invariant mass distributions; without taking spin correlations into account, all panels in Fig. 3 would be identical. Comparing the final distributions in the lower panels an asymmetry between positively and negatively charged leptons,

$$
\mathcal{A}=\frac{\ell^{+}-\ell^{-}}{\ell^{+}+\ell^{-}}
$$

is predicted indeed [5]. [The asymmetry is washed out somewhat in $p p$ collisions at the LHC where also anti-squarks $\tilde{q}^{*}$ are produced; see section 3.2 . Fig. 4 shows the parton-level asymmetry for the $\tilde{q}_{L}$ decay chain in SPS1a'. In particular for high invariant masses the dominance of positively charged leptons is evident. The NLO SQCD radiative corrections included in our decay analysis have a very small influence on the asymmetry. This is evident from the lower panel of Fig. 3, near the kinematic endpoint where the corrections to the invariant mass distribution of the positively charged lepton are biggest, the asymmetry is close to unity and changes are marginal as a result. 


\subsection{Squark cascade decays in $p p$ collisions at the LHC}

The more detailed numerical analysis presented in this final section is also based on the reference point SPS1a' [12] in the mSUGRA scenario. This point is located in the transition zone from the bulk to the coannihilation region in the $\left[M_{1 / 2}, M_{0}\right]$ gaugino/scalar mass plane. It corresponds to the region of maximum probability in the analysis of electroweak precision observables [30,31], driven by the contribution to the muon anomalous magnetic moment, $g_{\mu}-2$; the reference point is compatible with the WMAP measurement of the density of cold dark matter. The parameters of this mSUGRA scenario are defined as follows: gaugino mass $M_{1 / 2}=250 \mathrm{GeV}$, scalar mass $M_{0}=70 \mathrm{GeV}$, and trilinear coupling $A_{0}=-300 \mathrm{GeV}$ at the unification scale; moreover $\tan \beta=10$ and $\operatorname{sign}(\mu)=+$. After evolution down to the Terascale the squark, gluino, slepton, and neutralino masses that will be used in the phenomenological analysis are collected in Table 1. While Suspect [32] has been employed for the computation of the entries in the table, the Spheno [33] and SOFTSUSY [34] spectra are quite similar, with differences at the level of $1 \%$ and less.

Table 1 also presents the branching ratios which are based on NLO calculations as implemented in Susyhit [35] / Sdecay [36]. The $L$-squarks decay predominantly into the charginos $\tilde{\chi}_{1}^{ \pm}$and the neutralino $\tilde{\chi}_{2}^{0}$. $R$-squarks decay almost exclusively into the $\tilde{\chi}_{1}^{0}$ LSP which is bino-like. Hence, almost all of the squarks decaying to $\tilde{\chi}_{2}^{0}$ are indeed $L$-type squarks. Only a small admixture of $R$-type squarks diminishes the observed spin correlation and the asymmetry, Eq. (3.5), generated by the $\tilde{\chi}_{2}^{0}$ decay chain. More than half (i.e. $57 \%)$ of the neutralinos $\tilde{\chi}_{2}^{0}$ decay visibly into charged slepton-lepton-pairs. Since left-handed sleptons of the first two generations are heavy, the decays are dominated $(\mathrm{BR}=0.527)$ by decays into mixed $\tilde{\tau}_{1}$ states [37] with a reduced mass eigen-value, but still a significant fraction decays to $\tilde{e}_{R}, \tilde{\mu}_{R}$ (BR $=0.024$ for each mode). If the $e, \mu$ leptonic decays are used as experimental signals, the transverse momenta of the cascading $\tau \rightarrow e, \mu$ final states are strongly reduced. A large fraction of the $\tau \rightarrow e, \mu$ decay events will thus be lost in experimental cuts, about $90 \%$ for a transverse momentum cut of $10 \mathrm{GeV}$.

Since experiments will not distinguish quark from anti-quark jets, experimentally observable distributions will also receive contributions from anti-squark decay chains. For anti-squarks, by $C P$-invariance, the rôle of positively and negatively charged leptons is interchanged and the asymmetry $\mathcal{A}$ is reduced as a result. At the Tevatron, the equal rate for squark and anti-squark production even drives $\mathcal{A}$ to zero. However, the (non- $C P$-invariant) proton-proton initial state at the LHC ensures that more squarks than anti-squarks are produced and a non-zero asymmetry is predicted.

At the LHC, squarks are produced either directly in quark/anti-quark/gluon collisions, or they are decay products of gluinos as $M_{\tilde{g}}>M_{\tilde{q}}$ for the SPS1a' reference point. The production cross sections for both species have been determined including the next-to-leading order corrections of Ref. [38] as implemented in Prospino [39]. The NLO calculation of Ref. [38] has been performed for mass-degenerate 


\begin{tabular}{|c|c|c|c|c|c|}
\hline & $\operatorname{Mass}[\mathrm{GeV}]$ & $\operatorname{BR}\left([\tilde{g} \rightarrow] \tilde{q} \rightarrow \tilde{\chi}_{0}^{2}\right)[\%]$ & & $\operatorname{Mass}[\mathrm{GeV}]$ & $\operatorname{BR}\left(\tilde{\chi}_{2}^{0} \rightarrow \tilde{l}\right)[\%]$ \\
\hline$\tilde{u}_{L}$ & 558 & 32.2 & $\tilde{\chi}_{1}^{0}$ & 98 & \\
\hline$\tilde{d}_{L}$ & 564 & 31.6 & $\tilde{\chi}_{2}^{0}$ & 183 & $\tilde{e}_{R}^{+}: \quad 1.2$ \\
\hline$\tilde{u}_{R}$ & 542 & 0.6 & & & $\tilde{\tau}_{1}^{+}: \quad 26.4$ \\
\hline$\tilde{d}_{R}$ & 542 & 0.6 & $\tilde{e}_{R}$ & 124 & \\
\hline$\tilde{g}$ & 605 & $\begin{array}{ll}\tilde{u}_{L} / \tilde{d}_{L}: & 0.8 / 0.6 \\
\tilde{u}_{R} / \tilde{d}_{R}: & 0.03 / 0.03\end{array}$ & $\tilde{\tau}_{1}$ & 108 & \\
\hline
\end{tabular}

Table 1: Masses and decay branching ratios most relevant for evaluating the SPS1a' squark/gluino cascades.

$L$ and $R$-squarks and mass-degenerate first and second generation squarks of up and down type. Since the mass differences are in general small, in particular for the spectrum of the SPS1a' reference point where $\left[M_{L}^{2}-M_{R}^{2}\right] /\left[M_{L}^{2}+M_{R}^{2}\right] \approx 4 \%$, this is a valid approximation. In our tree-level calculation, to which we apply the K-factors, the exact kinematics is used. For the parton densities the CTEQ5 set [40] has been adopted and the renormalization and factorization scales have been set to the average mass of the produced particles. The production cross sections are displayed for squarks and anti-squarks of the first two generations in Table 2. The $p p$ initial state at the LHC clearly favours the direct production of squarks over the direct production of anti-squarks. Though equal numbers of squarks and anti-squarks are generated by gluino decays, the ratio of the number of anti-squarks over squarks is only slightly diminished because the branching ratio of gluinos into first and second generation $L$-squarks is small.

Table 2] also includes the cross sections times branching ratios $\sigma(\tilde{q}) \times \operatorname{BR}\left(\tilde{q} \rightarrow \chi_{2}^{0}\right)$ and $\sigma(\tilde{g}) \times \operatorname{BR}(\tilde{g} \rightarrow$ $\tilde{q} \rightarrow \chi_{2}^{0}$ ) for neutralino $\tilde{\chi}_{2}^{0}$ production from squark decays, and the final cross sections times branching ratios for the signal $\sigma[e, \mu]$, i.e. lepton production from squark cascades, with $\tau$ decays separated. With a nominal integrated luminosity of $300 \mathrm{fb}^{-1}$, a sample of roughly $2 \cdot 10^{5}$ events is accumulated for the SPS1a' benchmark for $e, \mu$ in the first two generations and, before experimental cuts, an initial number of $1.5 \cdot 10^{5} \tau$ events decaying leptonically into either electrons or muons. Although these calculations are of purely theoretical nature, they provide nevertheless a solid platform for estimating expectations before experimental simulations will finally include selection cuts, efficiencies etc.

Using these estimates of production cross sections and branching ratios, the invariant mass distributions for the SPS1a' decay chain can be calculated. Fig. 5 displays the LO and NLO [jet $\ell^{ \pm}$] invariant mass spectrum for positively and negatively charged leptons as predicted for the LHC. The distributions are normalized to the signal cross sections and are shown separately for $\tilde{\chi}_{2}^{0}$ decays to first/second generation leptons/sleptons and for $\tau / \tilde{\tau}$ decaying leptonically. $L / R$ mixing of the $\tau$ sleptons and the $\tilde{\chi}_{2}^{0}$ higgsino component exacerbate the analysis of the $\tau / \tilde{\tau}$ channel considerably; all these effects have been 


\begin{tabular}{|c|c|c||r|c|}
\hline & $\sigma(p p \rightarrow \tilde{q} / \tilde{g})[\mathrm{pb}]$ & $\sigma\left(\tilde{q} / \tilde{g} \rightarrow \tilde{\chi}_{2}^{0}\right)[\mathrm{pb}]$ & $\sigma\left(\tilde{l}_{R}\right)[\mathrm{pb}]$ & $\sigma(e, \mu)[\mathrm{pb}]$ \\
\hline \hline$\tilde{u}_{L} / \tilde{u}_{R}$ & $14.4 / 15.4$ & $4.6 / 0.1$ & $\tilde{e}_{R}+\tilde{\mu}_{R}: 0.57$ & 0.57 \\
$\tilde{d}_{L} / \tilde{d}_{R}$ & $6.7 / 7.4$ & $2.1 / 0.05$ & $\tilde{\tau}_{1}: 6.37$ & 0.39 \\
\hline$\tilde{u}_{L}^{*} / \tilde{u}_{R}^{*}$ & $1.7 / 1.9$ & $0.6 / 0.01$ & & \\
$\tilde{d}_{L}^{*} / \tilde{d}_{R}^{*}$ & $2.0 / 2.3$ & $0.6 / 0.01$ & & \\
\hline$\tilde{c}_{L} / \tilde{c}_{R}$ & $0.8 / 0.9$ & $0.3 / 0.01$ & & \\
$\tilde{s}_{L} / \tilde{s}_{R}$ & $1.2 / 1.4$ & $0.4 / 0.01$ & & \\
\hline$\tilde{c}_{L}^{*} / \tilde{c}_{R}^{*}$ & $0.9 / 1.0$ & $0.3 / 0.01$ & & \\
$\tilde{s}_{L}^{*} / \tilde{s}_{R}^{*}$ & $1.3 / 1.4$ & $0.4 / 0.01$ & & \\
\hline$\tilde{g}$ & 45.1 & 2.6 & & \\
\hline
\end{tabular}

Table 2: Cross sections for the production of squarks of the first two generations and gluino production in pp collisions at the $L H C$, and the production cross sections for the neutralino $\tilde{\chi}_{2}^{0}$ in the cascades. Also shown are the cross sections for R-sleptons after summing up all cascade channels. The resulting cross sections for electron/muon pairs from direct electron/muon production in the decay chain and from tau decays are collected in the right-most column.

taken into account in the numerical analysis. However, the leptonic $\tau$ decay signal is strongly reduced to a level of $10 \%$ if a transverse momentum cut of $10 \mathrm{GeV}$ is applied as indicated by the dotted lines. [For simplicity, the cut is applied in the squark rest frame. This approximation is adequate since squarks are produced predominantly at small velocities.] The experimentally observable distributions are clearly distinct despite of the anti-squark admixture to the squark sample. As a result of the anti-squark admixture, the $\ell^{+} / \ell^{-}$asymmetry (3.5) is slightly reduced at the LHC compared with Figure 4 for pure $\tilde{q}_{L}$ decays, with marginal NLO corrections as before. However, the asymmetry is still quite significant for large [jet $\ell]$ invariant masses as demonstrated in Refs. [5-7], even after including detector effects.

\section{Conclusions}

Edges/thresholds, distributions and correlations of particles in cascades play an important rôle in determining properties of supersymmetric particles, i.e. masses and spins. Lepton-lepton and jet-lepton invariant mass distributions are particularly useful instruments in this context.

In the present article we have studied how the parton final states in $\tilde{q} \rightarrow q \tilde{\chi}$ decays are affected by super-QCD corrections at next-to-leading order, in particular by the radiation of gluons in the decay process. The corrections modify the energy as well as the polarization of the particles. By performing an analytic next-to-leading order analysis we have provided a detailed understanding of origin and 
Jet-lepton invariant mass $\mathrm{d} \sigma / \mathrm{d} M_{\hat{q} l}[\mathrm{fb} / \mathrm{GeV}]$
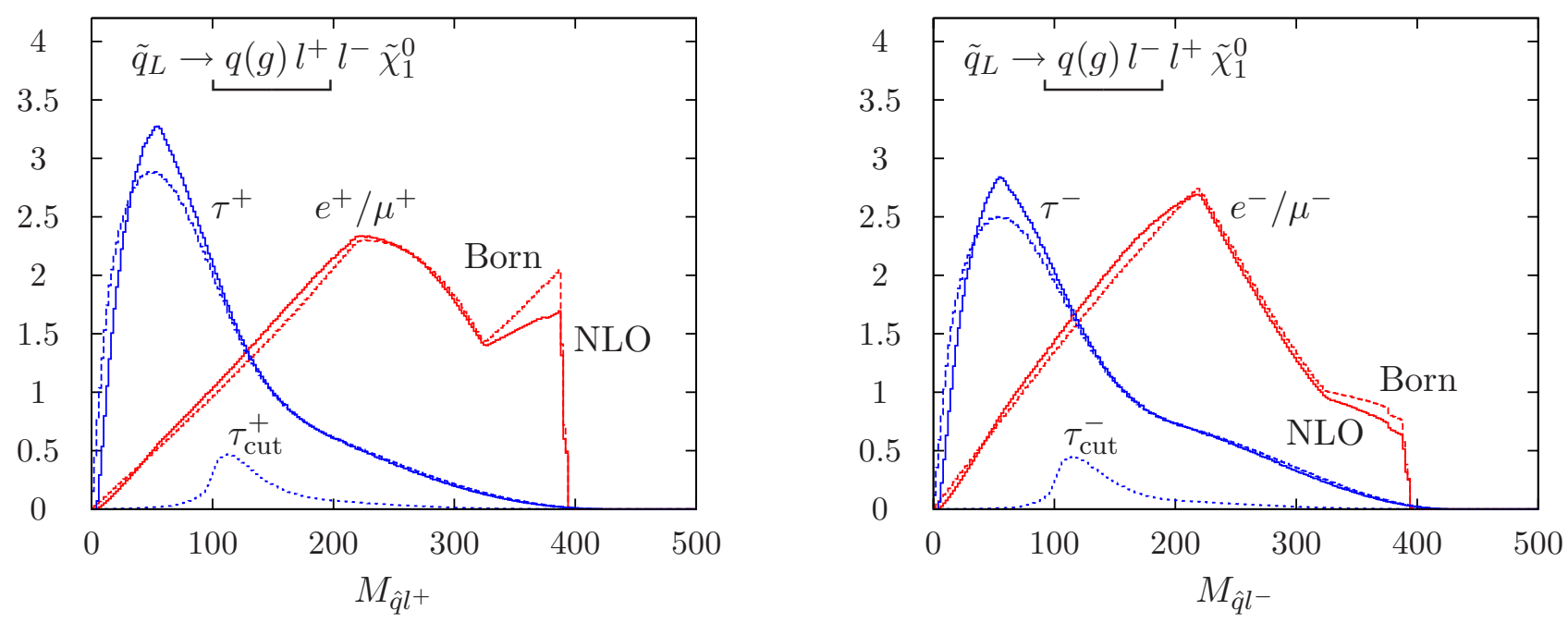

Figure 5: Invariant $[\hat{q} l]$ mass distributions, $y_{\mathrm{c}}=0.01$, for muons and electrons produced directly in the neutralino and slepton decay. For muon and electron pairs produced from tau decays in the decay chain, the reduction of the NLO cross sections for a transverse momentum cut of $10 \mathrm{GeV}$ is indicated by the dotted lines.

size of these effects. Moreover, we have studied particular decay channels and [jet $\left.\ell^{ \pm}\right]$invariant mass distributions, exemplified for the SPS1a' benchmark scenario, in $\tilde{q}_{L} \rightarrow q \ell^{+} \ell^{-} \tilde{\chi}_{1}^{0}$ decays. We have found significant gluonic corrections to the shape of the invariant [jet $\left.\ell^{+}\right]$mass distribution near the pronounced edge at the kinematic endpoint.

\section{Acknowledgments}

We gratefully acknowledge communications with G. Polesello, and D.J. Miller, P. Osland and A.R. Raklev. We are thankful to A. Djouadi for comments on the manuscript. This work was supported in part by the DFG SFB/TR9 "Computational Particle Physics", and by the European Community's MarieCurie Research Training Network HEPTOOLS under contract MRTN-CT-2006-035505. PMZ thanks the LPT and the Institut für Theoretische Physik E for the warm hospitality extended to him at the Université Paris-Sud and at the RWTH Aachen.

\section{References}

[1] I. Hinchliffe, F. E. Paige, M. D. Shapiro, J. Soderqvist and W. Yao, Phys. Rev. D 55 (1997) 5520 arXiv:hep-ph/9610544. 
[2] B. C. Allanach, C. G. Lester, M. A. Parker and B. R. Webber, JHEP 0009 (2000) 004 arXiv:hep-ph/0007009.

[3] B. K. Gjelsten, D. J. Miller and P. Osland, JHEP 0412 (2004) 003 [arXiv:hep-ph/0410303].

[4] G. Weiglein et al. [LHC/LC Study Group], Phys. Rept. 426 (2006) 47 arXiv:hep-ph/0410364.

[5] A. J. Barr, Phys. Lett. B 596 (2004) 205 arXiv:hep-ph/0405052.

[6] J. M. Smillie and B. R. Webber, JHEP 0510 (2005) 069 arXiv:hep-ph/0507170.

[7] C. Athanasiou, C. G. Lester, J. M. Smillie and B. R. Webber, JHEP 0608 (2006) 055 arXiv:hep-ph/0605286.

[8] L. T. Wang and I. Yavin, JHEP 0704 (2007) 032 arXiv:hep-ph/0605296.

[9] B. K. Gjelsten, D. J. Miller, P. Osland and A. R. Raklev, AIP Conf. Proc. 903 (2007) 257 arXiv:hep-ph/0611259.

[10] R. Kitano and Y. Nomura, Phys. Rev. D 73 (2006) 095004 arXiv:hep-ph/0602096.

[11] D. J. Miller, P. Osland and A. R. Raklev, JHEP 0603 (2006) 034 arXiv:hep-ph/0510356, and private communication.

[12] B. C. Allanach et al., Eur. Phys. J. C 25 (2002) 113 arXiv:hep-ph/0202233.

[13] J. A. Aguilar-Saavedra et al., Eur. Phys. J. C 46 (2006) 43 arXiv:hep-ph/0511344.

[14] W. Beenakker, R. Höpker and P. M. Zerwas, Phys. Lett. B 378 (1996) 159 arXiv:hep-ph/9602378.

[15] K. i. Hikasa and Y. Nakamura, Z. Phys. C 70 (1996) 139 [Erratum-ibid. C 71 (1996) 356] arXiv:hep-ph/9501382.

[16] S. Kraml, H. Eberl, A. Bartl, W. Majerotto and W. Porod, Phys. Lett. B 386 (1996) 175 arXiv:hep-ph/9605412.

[17] A. Djouadi, W. Hollik and C. Jünger, Phys. Rev. D 55 (1997) 6975 arXiv:hep-ph/9609419.

[18] W. Beenakker, R. Höpker, T. Plehn and P. M. Zerwas, Z. Phys. C 75 (1997) 349 arXiv:hep-ph/9610313.

[19] S. Y. Choi, A. Djouadi, M. Guchait, J. Kalinowski, H. S. Song and P. M. Zerwas, Eur. Phys. J. C 14 (2000) 535 arXiv:hep-ph/0002033.

[20] S. Y. Choi, J. Kalinowski, G. A. Moortgat-Pick and P. M. Zerwas, Eur. Phys. J. C 22 (2001) 563 [Addendum-ibid. C 23 (2002) 769] arXiv:hep-ph/0108117 and arXiv:hep-ph/0202039. 
[21] G. Schierholz, Proceedings, Quantum Chromodynamics, SLAC Summer Institute 1979.

[22] P. Binetruy, Phys. Lett. B 91 (1980) 245.

[23] S. Catani, G. Turnock, B. R. Webber and L. Trentadue, Phys. Lett. B 263 (1991) 491.

[24] T. Plehn, H. Spiesberger, M. Spira and P. M. Zerwas, Z. Phys. C 74 (1997) 611 arXiv:hep-ph/9703433.

[25] P. Richardson, JHEP 0111 (2001) 029 arXiv:hep-ph/0110108.

[26] G. Sterman and S. Weinberg, Phys. Rev. Lett. 39 (1977) 1436.

[27] A. Heister et al. [ALEPH Collaboration], Eur. Phys. J. C 35 (2004) 457.

[28] N. Kauer, arXiv:0708.1161 [hep-ph].

[29] M. Drees, W. Hollik and Q. Xu, JHEP 0702 (2007) 032 arXiv:hep-ph/0610267.

[30] B. C. Allanach, K. Cranmer, C. G. Lester and A. M. Weber, JHEP 0708 (2007) 023 arXiv:0705.0487 [hep-ph]].

[31] J. R. Ellis, S. Heinemeyer, K. A. Olive, A. M. Weber and G. Weiglein, JHEP 0708 (2007) 083 arXiv:0706.0652 [hep-ph]].

[32] A. Djouadi, J. L. Kneur and G. Moultaka, Comput. Phys. Commun. 176 (2007) 426 arXiv:hep-ph/0211331.

[33] W. Porod, Comput. Phys. Commun. 153 (2003) 275 arXiv:hep-ph/0301101.

[34] B. C. Allanach, Comput. Phys. Commun. 143 (2002) 305 arXiv:hep-ph/0104145.

[35] A. Djouadi, M. M. Mühlleitner and M. Spira, Acta Phys. Polon. B 38 (2007) 635 arXiv:hep-ph/0609292.

[36] M. Mühlleitner, A. Djouadi and Y. Mambrini, Comput. Phys. Commun. 168 (2005) 46 arXiv:hep-ph/0311167.

[37] S. Y. Choi, K. Hagiwara, Y. G. Kim, K. Mawatari and P. M. Zerwas, Phys. Lett. B 648 (2007) 207 arXiv:hep-ph/0612237.

[38] W. Beenakker, R. Höpker, M. Spira and P. M. Zerwas, Nucl. Phys. B 492 (1997) 51 arXiv:hep-ph/9610490.

[39] The Prospino computer code can be obtained from http://www.ph.ed.ac.uk/ tplehn/prospino/ or from http://people.web.psi.ch/spira/prospino/.

[40] H. L. Lai et al. [CTEQ Collaboration], Eur. Phys. J. C 12 (2000) 375 arXiv:hep-ph/9903282. 